\title{
$\alpha$-Actinin-4 Gene Mutations, Steroid Responsiveness and FSGS in Adult Onset-Nephrotic Syndrome
}

\author{
Asra Tabassum Fatima Jaffer ${ }^{* 1}$, Sree Bhushan Raju ${ }^{2}$ and Parveen Jahan ${ }^{3}$ \\ ${ }^{1}$ Osmania University, Hyderabad, Telangana, India \\ ${ }^{2}$ Department of Nephrology, Nizam's Institute of Medical Sciences, Hyderabad, India
}

${ }^{3}$ Department of Zoology, School of Life Sciences, MANUU, Hyderabad, India

*Corresponding author: Jaffer ATF, Research Associate, Osmania University, Hyderabad, Telangana, India, Tel: 040 2768 2363; E-mail: tabassum27asra@gmail.com Rec Date: 27 Mar, 2016; Acc Date: 16 Apr, 2016; Pub Date: 25 Apr, 2016

Copyright: @ 2016 Jaffer ATF, This is an open-access article distributed under the terms of the Creative Commons Attribution License, which permits unrestricted use, distribution, and reproduction in any medium, provided the original author and source are credited.

\begin{abstract}
Focal segmental glomerulosclerosis (FSGS) is a common pattern of injury seen in primary as well as secondary renal disorders and is a major cause of steroid- resistant nephrotic syndrome (SRNS) and end-stage kidney disease (ESKD). SRNS is defined by resistance to standard steroid therapy and it remains one of the most intractable causes of kidney failure. Mutations in a-actinin-4 gene (ACTN4) represent a frequent cause of SRNS associated with an adult onset form of FSGS. We screened a cohort of 374 subjects from the South Indian population for the presence of two missense mutations, K255E and S262P in exon 8 of ACTN4 gene encoding $\alpha$-actinin-4, an actinfilament crosslinking and bundling protein. Our results revealed that these two mutations were seen only among the patients $(4 \%)$ in heterozygous form and absent in the controls suggesting that, these mutations were found to be disease causing in nature and the regulation of the actin cytoskeleton of glomerular podocytes may be altered in the patients. Further categorization revealed that K255E and S262P mutations were together responsible for $5 \%$ of SRNS and $15 \%$ of FSGS cases in our study group. Conversely, these mutations were not found in the controls as well as in the patients with steroid-sensitive nephrotic syndrome (SSNS). This is the first report from India, more studies are warranted to establish the frequency of ACTN4 mutations in our population and such analysis may help in developing mutation(s) specific therapeutic interventions in future.
\end{abstract}

Keywords: a-Actinin-4; ACTN4 mutations; Steroid resistant nephrotic syndrome; Focal segmental glomerulosclerosis; ER stress

\section{Introduction}

Podocytes are terminally differentiated cells that line the outer aspects of the glomerular capillaries. The highly ordered podocyte architecture consists of a cell body from which emerge major processes, which branch into foot processes that interdigitate with those of neighbouring podocytes to provide the structural platform upon which a molecular sieve is formed. The foot processes are endowed with a microfilament-based contractile apparatus composed of actin, myosin-II, $\alpha$-actinin, talin, paxillin, and vinculin, and are anchored to the glomerular basement membrane via an a3b1-integrin complex. The intricate morphology of the podocyte, coupled to its exposure to distensile forces within the glomerular capillary render these cells susceptible to damage in many nephropathies, including idiopathic nephrotic syndrome (INS). The underlying histopathology in INS may be minimal change disease (MCD), mesangioproliferative glomerulo nephritis (MPGN) or focal segmental glomerulo sclerosis (FSGS) [1].

Corticosteroid therapy remains the gold standard treatment and based on the response to steroid therapy NS may be classified as steroid sensitive nephrotic syndrome (SSNS) and steroid-resistant nephrotic syndrome (SRNS), with the possibility that there is a different pathophysiology which confers response to steroids [2]. However, this does not fully explain why some patients who are initially responsive later become resistant. Most genetic forms of SRNS represent structural changes in the glomerulus or, more specifically, the podocyte, which are unlikely to respond to current therapy. On the whole, this only explains a small percentage of total cases. It is reported that approximately $20 \%$ of the cases are steroid resistant clinically, and of these $60 \%$ are biopsy-proven FSGS, a term which is in practice interchangeably used with SRNS [3].

FSGS is a common glomerular lesion and a significant cause of endstage renal disease. Clinically, FSGS patients present with variable levels of proteinuria and a progressive loss of renal function. Pathologically, FSGS is characterized by segmental sclerosis in a proportion of glomeruli, the filtering units of the kidney. Accumulating evidence suggests that defects in podocytes initiate processes leading to the degeneration of filtration integrity and the development of sclerotic lesions $[1,3]$.

a-Actinin- 4 is a member of the actinin protein family that consists of an actin binding domain in the $\mathrm{N}$ terminus, four spectrin-like repeats in the central region, and two EF-hand motifs in the $\mathrm{C}$ terminus. Although both $a$-actinin- 1 and -4 are expressed in mouse podocytes, $a$-actinin- 4 is the sole member of the actinin family expressed in human podocytes. $\alpha$-Actinin- 4 is widely expressed in mammalian tissues and organs. However, despite the widespread expression, podocytes appear to be the primary site of manifestations of diseases induced by genetic alterations of ACTN4 (19q 13.2), the gene that encodes $a$-actinin-4. How do alterations of ACTN4 cause podocyte damage? A subset of FSGS2-associated a-actinin-4 mutations were found in the actin-binding domain and shown to increase actin binding and aggregation of $a$-actinin- 4 consequently leading to podocyte damage [4]. 
Page 2 of 4

The characteristic feature of ACTN4 mutations is slow progressive form of kidney dysfunction associated with mild to moderate proteinuria, renal insufficiency with slow progression to end stage renal disease, and focal segmental glomerulosclerotic pattern of tissue injury. Previously, about ten missense mutations have been described in ACTN4 that co-segregate with disease in families of western European and African ancestry exhibiting autosomal dominant pattern of inheritance. These mutations increase the affinity of ACTN4 to actin filaments (F-action) as well as alter their intracellular localization. The underlying disease mechanism is not entirely clear, but studies to date are consistent with a gain-of-function model [3]. Abrogation of $\alpha$ actinin-4 expression in mice was shown to yield severe glomerular disease. Furthermore, recent investigations suggest that the familial mutations promote $\alpha$-actinin- 4 aggregation and thereby target the protein for degradation via the proteasome pathway, resulting in a partial loss-of-function. In support of the former mechanism, the severity of the FSGS-like phenotype correlates directly with mutant $\alpha$ actinin-4 levels in transgenic mice. Thus, it remains unclear whether and how these two viewpoints on gain-of-function and loss-offunction can be reconciled [1].

INS is a clinically and genetically heterogeneous entity. Mutations in NPHS2, a slit-diaphragm protein, cause a recessively inherited form of nephrotic syndrome of widely variable age of onset and disease severity. Mutations in the TRPC6 channel because an autosomal dominant form of FSGS that is phenotypically similar to the ACTN4associated form of disease [3]. There are no reports from the Indian population with regard to mutation analysis of the ACTN4 gene; this is the first study that attempts to screen for selected ACTN4 exon 8 mutations among adult-onset nephrotic syndrome patients from the Asian Indian population.

\section{Materials and Methods}

A total of 374 subjects (124 patients and 250 controls) were recruited in the present study. After an informed consent, the patients were recruited from Nizam's Institute of Medical Sciences and Hospital, Hyderabad, India, on being confirmed as nephrotics by the consultant nephrologist based on clinical, laboratory investigations and those who were already on corticosteroid therapy at the time of the study. Subjects diagnosed with secondary nephrotic syndrome, lupus nephritis, renal failure and other associated conditions were excluded from the study.

Information relating to the age, gender, family history, consanguinity was collected from the total cohort and additional information relating to age at disease onset, histopathology and responsiveness to steroid therapy were collected from the patient group. The criterion to distinguish between SRNS and SSNS; and the histopathological variants are given elsewhere [5].

$5 \mathrm{ml}$ of venous blood was collected in EDTA vaccutainers from both the patient and control groups and DNA was isolated using the nonenzymatic method [6]. Genetic analysis of the selected mutations was done by tetra primer ARMS PCR [7]. PCR amplification of genomic DNA was performed with 100ng of human DNA in $10 \mu \mathrm{PCR}$ reaction mix containing $1 \mu \mathrm{l} 10 \mathrm{X}$ PCR buffer $(100 \mathrm{mM}$ Tris $\mathrm{HCl}, \mathrm{pH}=8.8,500$ $\mathrm{mM} \mathrm{KCl}, 15 \mathrm{mM} \mathrm{MgCl} 2,0.01 \% \mathrm{w} / \mathrm{v}$ gelatin); $200 \mu \mathrm{M}$ of each dNTPs; 25 pmol of each primer and $0.5 \mathrm{U}$ Taq polymerase. The primers were designed using Primer3, an online primer designing tool. The primers used for A763G were outer forward 5' AAGGATGGTCTTGCCTTCAATGCCCTGA3', outer reverse
5'ACTCCAGGAGGTCGCTGGCCAGCTT3', inner forward 5'AACACGGCCCGGCCCGACGCGA3', and inner reverse 5'GCTGGACACATAGGTCATTATGGCATC3', while the primers for the second mutation, T784C were: outer forward 5'CAGACCAGAGCTGATTGAGTATGACA3', outer reverse 5'GCTGCATCTCCTGGATAGTCTTTTG3', inner forward 5'GAAGGCCATAATGACCTATGGGC3', inner reverse 5'CTGAAAAGGCATGGTAGAAGCTTGA3', respectively. The PCR products were run on $2 \%$ agarose gel for $20 \mathrm{~min}$ to confirm amplification and record the genotypes. Analysis of the amplified DNA generated fragments of various sizes corresponding to different alleles was done by electrophoresis. Based on the number of bands and fragment sizes visualized, the samples were genotyped as homozygotes or heterozygotes for the mutations studied (Figures 1a - 1c). Descriptive statistics were obtained using SPSS software (version 20.0). The allele and genotype frequencies for Hardy Weinberg equilibrium were calculated, $\mathrm{p}$-value less than 0.05 was considered as statistically significant. An SNP prediction algorithm Polymorphism phenotyping (PolyPhen-2) was used to predict the effect of the selected mutations on protein function in the present study. PolyPhen-2 is an automatic tool for prediction of possible impact of an amino acid substitution on the structure and function of a human protein based on a number of features comprising the sequence, phylogenetic and structural information characterizing the substitution.

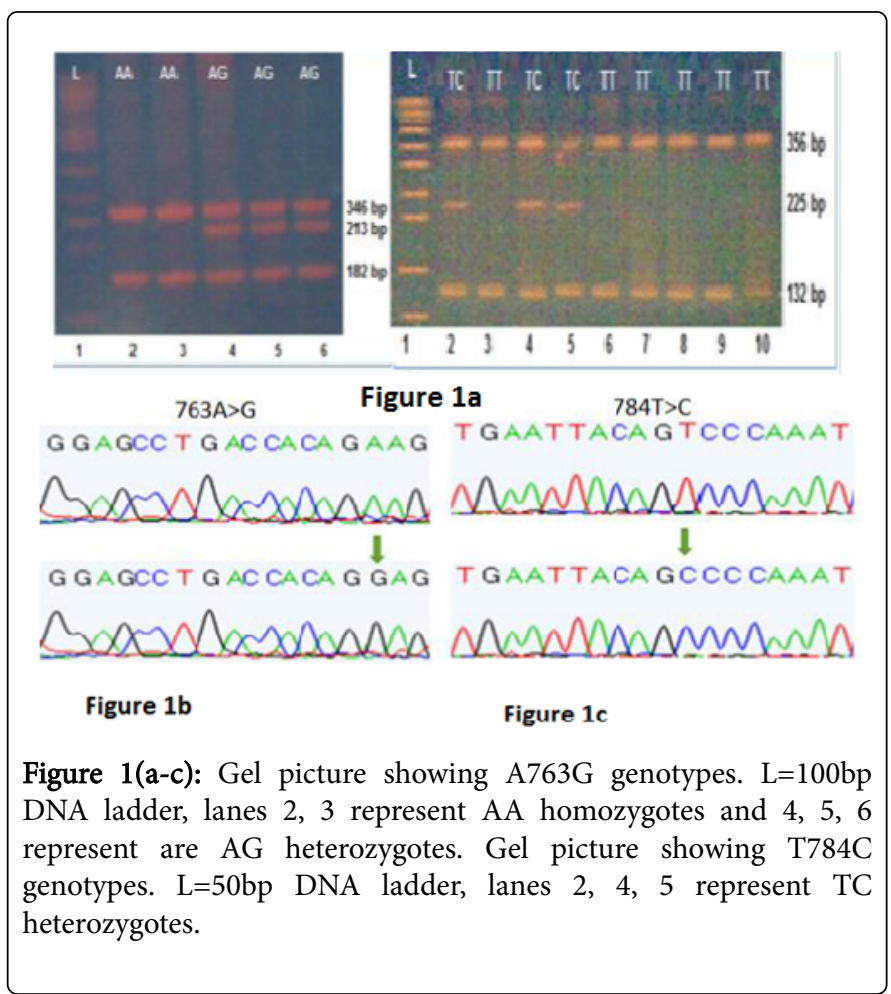

\section{Results and Discussion}

To evaluate the genotype-phenotype correlation among patients with INS, a cohort of 374 were examined for selected mutations in the exon 8 of ACTN4 gene. The mean age of INS patients was $26.38 \pm 8.14$ years and that of the controls was $32.16 \pm 8.5$ years respectively. Of the 124 INS patients $80.6 \%$ were SRNS and $19.3 \%$ were SSNS. Kidney biopsy showed MCD in $45 \%$, MPGN in $37 \%$ and FSGS in $41 \%$, the male to female ratio was 1.9:1 in the patient group. Mutational analyses 
at two hot-spot mutations at exon 8 of $\alpha$-actinin 4 showed no relevant alterations in majority of the patients studied.

The A763G substitution in the exon 8 of ACTN4 gene leads to a missense mutation K255E [8], this mutation was detected in heterozygous form in $2.4 \%$ of the INS patients and in none of the controls. The overall genotype frequencies were $97.5 \%$ for $\mathrm{AA}$ and $2.4 \%$ for AG. Neither the cases nor the controls were of GG genotype. Further analysis was carried in relation to steroid response and the frequency of the genotypes AA and AG was $97 \%$ and $3 \%$ respectively in the SRNS group, on the other hand all the SSNS patients were of AA genotype.

The second mutation studied, was a T784C substitution in the ACTN4 gene that leads to a missense mutation S262P [8]. About 1.6\% of the INS patients exhibited S262P mutation in heterozygous condition and none of the controls had it. The frequencies of TT and TC genotypes were $98.3 \%$ and $1.6 \%$ respectively; the CC genotype was not observed in either patients or controls. Analysis in relation to steroid response revealed $98 \%$ and $2 \%$ of TT and TC genotypes correspondingly in the SRNS group, whereas all of the SSNS patients had TT genotype. Interestingly it was observed that the A763G and T784C mutations accounted for 5\% of all the SRNS cases and $15 \%$ of the patients with FSGS (Figure 2). No deviation was observed in the Hardy Weinberg Equilibrium with respect to genotypes for both the mutations in the control as well as the patient groups $(p>0.05)$. Polyphen-2 predicted both the studied substitutions to be possibly damaging / pathogenic mutations with a PSIC score difference of 1.56 and 1.86 respectively (Table 1 ).

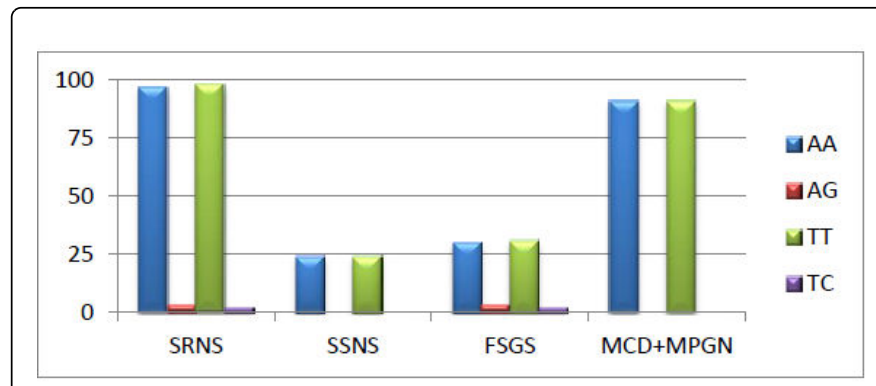

Figure 2: Distribution of A763G and T784C genotypes among cases with respect to response and histopathology.

\begin{tabular}{|c|c|c|c|c|c|c|c|c|c|c|}
\hline \multirow{2}{*}{ Category } & \multicolumn{3}{|c|}{ A763G Genotypes (\%) } & \multicolumn{2}{|c|}{ Allele frequency } & \multicolumn{3}{|c|}{ T784C Genotypes (\%) } & \multicolumn{2}{|c|}{ Allele frequency } \\
\hline & $\mathrm{AA}$ & $A G$ & GG & A & G & TT & TC & $\mathrm{CC}$ & $\mathrm{T}$ & $\mathrm{C}$ \\
\hline $\begin{array}{l}\text { Control } \\
250\end{array}$ & $\begin{array}{l}250 \\
(100)\end{array}$ & $\begin{array}{l}0 \\
(0)\end{array}$ & $\begin{array}{l}0 \\
(0)\end{array}$ & 1 & 0 & $\begin{array}{l}250 \\
(100)\end{array}$ & $\begin{array}{l}0 \\
(0)\end{array}$ & $\begin{array}{l}0 \\
(0)\end{array}$ & 1 & 0 \\
\hline $\begin{array}{l}\text { Case } \\
124\end{array}$ & $\begin{array}{l}121 \\
(97.5)\end{array}$ & $\begin{array}{l}3 \\
(2.4)\end{array}$ & $\begin{array}{l}0 \\
(0)\end{array}$ & 0.99 & 0.01 & $\begin{array}{l}122 \\
(98.3)\end{array}$ & $\begin{array}{l}2 \\
(1.6)\end{array}$ & $\begin{array}{l}0 \\
(0)\end{array}$ & 0.99 & 0.01 \\
\hline
\end{tabular}

Table 1: Distribution of A763G and T784C genotypes among control and case groups.

The present findings suggest that INS is a heterogeneous disease, since mutations in ACTN4 explain approximately $4 \%$ of the condition in our studied population. None of the mutations studied were detected in SSNS patients whereas all the patients with mutations were SRNS; likewise mutations were detected only in patients with FSGS and not with MCD or MPGN. Thus, indicating that these mutations can be considered as markers of steroid resistance and FSGS.

It should be noted that, the ACTN4 mutations (K255E and S262P) selected in the present study, have been reported to be retained in the endoplasmic reticulum (ER), while the wild type $\alpha$-actinin4 localize in the plasma membrane in cultured cell based transfection studies $[9,10]$. Further disturbances in the efficiency of protein folding due to missense mutations and / or other ischemic insults may result in the accumulation of unfolded or misfolded proteins in the ER, leading to ER stress that may have the potential to induce cellular damage. The unfolded protein response (UPR), an adaptive response to ER stress, is initially aimed at compensating for damage but can eventually trigger cell death if ER dysfunction is severe or prolonged, which may partly explain the adult onset of kidney disease in patients harbouring ACTN4 mutations. This, in turn, may provide a possible pathomechanism for the loss of protein function in patients carrying missense mutations with defective intracellular transport to the plasma membrane [11]. It has now been reported that the intracellular mislocalization of K256E $\alpha$-actinin- 4 in mouse podocytes, undermines the processes of cell spreading and migration, and impairs the formation of actin-rich peripheral projections (K256E is the mouse mutant corresponding to human K255E). In addition studies suggest that such defects in key cytoskeletal-associated processes may compromise the podocyte's ability to cope with the demands of the glomerular environment, maintain foot processes structure, and thereby initiate the progression towards sclerosis [3]. Cultured podocytes from the mice adhere poorly to basement membrane components, suggesting that podocyte loss may contribute to podocytopenia and FSGS [12].

Considering the above explanations, FSGS may be associated with glomerular epithelial cell (GEC; podocyte) apoptosis due to acquired injury or mutations in $\alpha$-actinin-4 gene. It is reported that the $\alpha$ actinin-4 mutants retained in the ER intensify apoptosis and triggers several metabolic abnormalities, which may lead to cellular injury and glomerulosclerosis [9]. Such situations mean a death sentence, but there is still hope as promising therapeutic approaches using chemical chaperones have been shown to correct protein processing defects associated with several mutations of membrane proteins such as CFTR in cystic fibrosis $[13,14]$ and AQP2 in nephrogenic diabetes insipidus $[15,16]$. Thus, identifying such mutations may help in the development of mutation specific therapeutic interventions that may be beneficial in alleviating the disease condition. 
Citation: Jaffer ATF, Raju SB, Jahan P (2016) a-Actinin-4 Gene Mutations, Steroid Responsiveness and FSGS in Adult Onset-Nephrotic Syndrome. Hereditary Genet 5: 167. doi:10.4172/2161-1041.1000167

Page 4 of 4

\section{Conclusions}

The present study is the first report from the Asian Indian population that has identified probable disease causing missense mutations in a proportion of the studied subjects. It has been observed that about $4 \%$ of the NS cases were found to harbour either of the two selected mutations. Furthermore all of them were steroid resistant and carried the mutations in heterozygous condition showing the dominant nature of the allele that is responsible for susceptibility to the onset of NS and conferring steroid resistance. All these cases were found to be FSGS. We did not find any case carrying both mutations indicating that these mutations can independently be instrumental in the causation of adult onset SRNS/FSGS. Together K255E and S262P mutations do seem to play a major role in the pathogenesis of SRNS and FSGS as they account for $5 \%$ and $15 \%$ of the cases respectively in the present study group. This information can be further made use of in developing alternate and specific treatment regimens based on the kind of mutation(s) and their consequences at the cellular level. More studies are warranted in different ethnic groups with larger sample sizes in order to confirm our observations.

\section{Acknowledgement}

The authors wish to thank all the study subjects for their wilful participation and the medical staff in collecting the blood samples from the subjects. We are also grateful to the Indian Council of Medical Research (ICMR), New Delhi for providing financial assistance to AT. We thank Dr. K. Prasanna Latha for her valuable suggestions whilst critically evaluating the manuscript.

\section{References}

1. Michaud JL, Chaisson KM, Parks RJ, Kennedy CR (2006) FSGS associated $\alpha$-actinin-4 (K256E) impairs cytoskeletal dynamics in podocytes. Kidney Int 70:1054-1061.

2. Jaffer AT, Ahmed WU, Raju DS, Jahan P (2011) Foothold of NPHS2 mutations in primary nephrotic syndrome. J Postgrad Med 57: 314-320.

3. Weins A, Kenlan P, Herbert S, Le TC, Villegas I, et al. (2005) Mutational and biological analysis of alpha-actinin-4 in focal segmental glomerulosclerosis. J Am Soc Nephrol 16: 3694-3701.
4. Liu Z, Blattner SM, Tu Y, Tisherman R, Wang JH, et al. (2011) aActinin-4 and CLP36 Protein Deficiencies Contribute to Podocyte Defects in Multiple Human Glomerulopathies. J Biol Chem 286: 30795-30805.

5. Tabassum A, Rajeshwari C, Jahan P, Anuraj N, Mukesh Y (2014) Structural characterization and mutational assessment of Podocin - a novel drug target to nephrotic syndrome -an in silico approach. Interdiscip Sci Comput Life Sci 6:32-39.

6. Unnisa W, Tabassum A, Umme N, Mahwish, et al. (2012) Does disease status and steroid responsiveness in idiopathic nephrotic syndrome depend on ACE I/D gene polymorphism? A study from South India. Asian Journal of Biological Sciences 5: 148-156.

7. Ye S, Dhillon S, Ke X, Collins AR, Da IN (2001) An efficient procedure for genotyping single nucleotide polymorphisms. Nucleic Acids Res 29: E88-98.

8. Kaplan JM, Kim H, North KN, Rennke H, Correia LA, et al. (2000) Mutations in ACTN4, encoding alpha-actinin-4, cause familial focal segmental glomerulosclerosis. Nat Genet 24: 251-256.

9. Cybulsky AV, Takano T, Papillon J, Bijian K, Guillemette J, et al. (2009) Glomerular epithelial cell injury associated with mutant $\alpha$-actinin-4. Am J Physiol Renal Physiol 297:F987-F995.

10. Ohashi T, Uchida K, Uchida S, Sasaki S, Nihei H (2003) Intracellular mislocalization of mutant podocin and correction by chemical chaperones. Histochem Cell Biol 119: 257-264.

11. Inagi R (2009) Endoplasmic reticulum stress in the kidney as a novel mediator of kidney injury. Nephron Exp Nephrol 112: e1-e9.

12. Dandapani SV, Sugimoto H, Matthews BD, Kolb RJ, Sinha S, et al. (2007) Alpha -actinin-4 is required for normal podocyte adhesion. J Biol Chem 282:467-477.

13. Welsh MJ, Smith AE (1993) Molecular mechanisms of CFTR chloride channel dysfunction in cystic fibrosis. Cell $73: 1251-1254$.

14. Sato S, Ward CL, Krouse ME, Wine JJ, Kopito RR (1996) Glycerol reverses the misfolding phenotype of the most common cystic fibrosis mutation. J Biol Chem 271:635-638.

15. Tamarappoo BK, Verkman AS (1998) Defective aquaporin-2 trafficking in nephrogenic diabetes insipidus and correction by chemical chaperones. J Clin Invest 101:2257-2267.

16. Tamarappoo BK, Yang B, Verkman AS (1999) Misfolding of mutant aquaporin-2 water channels in nephrogenic diabetes insipidus. J Biol Chem 274:34825-34831. 